\title{
SECOND OPERATION FOR UPPER MEDIASTINAL LYMPHADENOPATHY AFTER COMPLETE RESECTION FOR CANCER OF THE LEFT LUNG
}

Masami Sato, MD, ${ }^{\mathrm{a}, \mathrm{b}}$ Teruaki Koike, MD, ${ }^{\mathrm{a}}$ Kohei Yokoi, MD, ${ }^{\mathrm{a}}$ Hirohito Tada, MD, ${ }^{\mathrm{a}}$ Motoyasu Sagawa, MD, ${ }^{\mathrm{a}, \mathrm{b}}$ Akira Sakurada, MD, ${ }^{\mathrm{b}}$ Tatsuo Tanita, MD, ${ }^{\mathrm{b}}$ Takashi Kondo, MD, ${ }^{\mathrm{a}, \mathrm{b}}$ Shigefumi Fujimura, MD, ${ }^{\mathrm{a}, \mathrm{b}}$ and Ryosuke Tsuchiya, MD, ${ }^{\mathrm{a}}$ Sendai, Japan

Lymphatic flow in cases of cancer of the left lung sometimes results in upper mediastinal lymphadenopathy. ${ }^{1}$ Some intensive trials ${ }^{2,3}$ of upper mediastinal node dissection combined with lobectomy of the left lung have been carried out in Japan. The prognosis of patients in these trials, however, was poor, as evidenced by a high mortality and morbidity.

On the other hand, in a few patients, metastasis was limited to the mediastinal nodes throughout the clinical course. We have a long-surviving patient who underwent such dissection 4 years after lobectomy of the left upper lobe. The clinical significance of the second operation for upper mediastinal lymphadenopathy after complete resection for left lung cancer is still unknown. Thus, in this study, we collected and analyzed the data on 10 similar cases in cooperation with the Japan Lung Cancer Surgical Study Group.

Clinical data on 10 patients who underwent dissection after lobectomy of the left lung were collected from 4 hospitals in Japan. In all patients, swelling of upper mediastinal nodes was not detected by chest computed tomography (CT). At the first operation, lobectomy with systematic hilar and mediastinal node dissection was performed through a posterolateral thoracotomy and then the patients were followed up. Lymphadenopathy of the upper mediastinum was detected by follow-up CT except in 1 patient. In this patient, who had involvement of the lower pretracheal node, an enlarged lymph node was diagnosed by chest CT 27 days after the first operation. Before the second operation, only 1 of the 10 patients had received chemotherapy (cisplatin and vindesine).

At the second operation, a median sternotomy was used for

From the Lung Cancer Surgical Study Group ${ }^{\mathrm{a}}$ of the Japan Cancer Oncology Group (JCOG), Japan, and the Department of Thoracic Surgery, ${ }^{\mathrm{b}}$ Institute of Development, Aging and Cancer (IDAC), Tohoku University, Sendai, Japan.

This study was partly supported by grants-in-aid from the Ministry of Education, Science, Sports and Culture of the Japanese Government.

Received for publication Oct 18, 1999; accepted for publication Jan 26, 2000.

Address for reprints: Masami Sato, MD, the Department of Thoracic Surgery, Institute of Development, Aging and Cancer, Tohoku University, 4-1 Seiryo-machi, Aoba-ku, Sendai 980-8575, Japan (E-mail: m-sato@idac.tohoku.ac.jp).

J Thorac Cardiovasc Surg 2000;119:1291-2

Copyright $\odot 2000$ by The American Association for Thoracic Surgery $0022-5223 / 2000 \$ 12.00+0 \quad \mathbf{1 2 / 5 4 / 1 0 6 0 3 9}$

doi: $10.1067 / \mathrm{mtc} .2000 .106039$ dissection of pretracheal nodes (No. 3), paratracheal nodes (No. 2), and the highest mediastinal nodes (No. 1). Histopathologic examinations revealed involvement of the lymph nodes in each patient. In 6 of the 10 patients, the involved nodes were completely resected. In the remaining 4 patients, however, the tumors were not removed because of extranodal invasion to the trachea or phrenic nerve.

After the second operation, 3 patients received adjuvant radiotherapy, and 1 underwent adjuvant radiotherapy combined with chemotherapy (cisplatin and vindesine). Five-year survival of all patients from the time of the first operation was $23 \%$. Fig 1 shows survival after the first operation according to the resectability at the second operation. Among the 4 patients in whom tumors were incompletely resected, there were no 5-year survivors. On the other hand, 5-year survival of the patients with complete resection was $44 \%$. In 3 of the 6 patients with complete resection, mediastinal lymphadenopathy had been detected more than 2 years after the first operation. The survival curve of these patients is shown in Fig 2 compared with that of 3 patients whose recurrence was detected within 2 years of the initial operation.

Follow-up since the first operation has revealed that recurrence was limited to lymphatic tissues in 4 patients. In the other 6 patients, however, hematogenous metastasis with lymphatic involvement was also detected after the second operation and resulted in death from cancer.

Metastasis to the upper mediastinal lymph nodes often occurs in patients with left lung cancer. ${ }^{1}$ Some intensive trials such as systematic bilateral mediastinal dissection combined with lobectomy performed through a median sternotomy for left lung cancer have failed to improve the prognosis in such cases. On the other hand, in a few patients, metastasis was limited to regional nodes throughout the clinical course until death from cancer. Indeed, in 4 of our 10 patients, recurrence was observed in only the regional nodes. This suggests that in some patients curative surgical resection is possible. In the present study, survivors after the second operation were those who had no detected recurrence for a long period after the first operation. Martini and colleagues ${ }^{4}$ reported that recurrence developed within the first 2 years after surgery in $60 \%$ of their patients who had a recurrence. They also mentioned that the sites of first recurrence were distant in most cases. ${ }^{4}$ Thus we speculate that most of the patients who survive more than 2 years after the first operation without distant metastasis but with regional recurrence belong to a highly limited subgroup with relatively weak or no biologic potential for distant metastasis. 


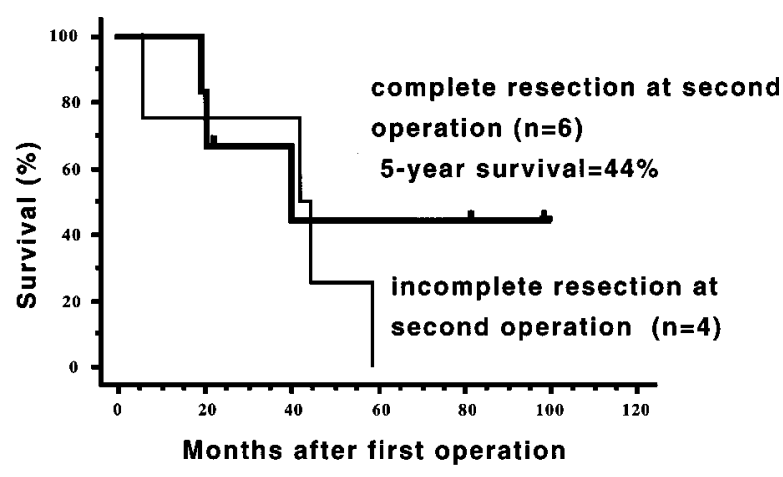

Fig 1. Survival curves after the first operation. There was no significant difference $(P=.46, \log$ rank test). The $95 \%$ confidence limits at 5 years in the group with complete resection were 0 to 0.88 .

Extensive dissection of upper mediastinal nodes combined with lobectomy requires much time and effort and is highly invasive. However, recurrence is frequent. In our present study, no major complications were observed, and there were no operative deaths.

The present study suggests that upper mediastinal node dissection through a median sternotomy might have a role in the treatment of selected patients who have survived more than 2 years since the first operation and who do not have distant metastasis but do have regional recurrence. Considering both the biologic behavior of the tumor and the invasiveness of this surgery, this approach may be an alternative treatment for selected recurrence of left lung cancer.

This study was carried out by the work in Lung Cancer Surgical Study Group (Chairman: Harubumi Kato) of JCOG (Chairman: Masanori Shimoyama). The cases in this study were collected from IDAC Hospital of Tohoku University (Head: Shigefumi Fujimura), Niigata Cancer Center Hospital (Teruaki Koike), Tochigi Cancer Center (Kohei Yokoi), and Osaka General Medical Center (Hirohito Tada).

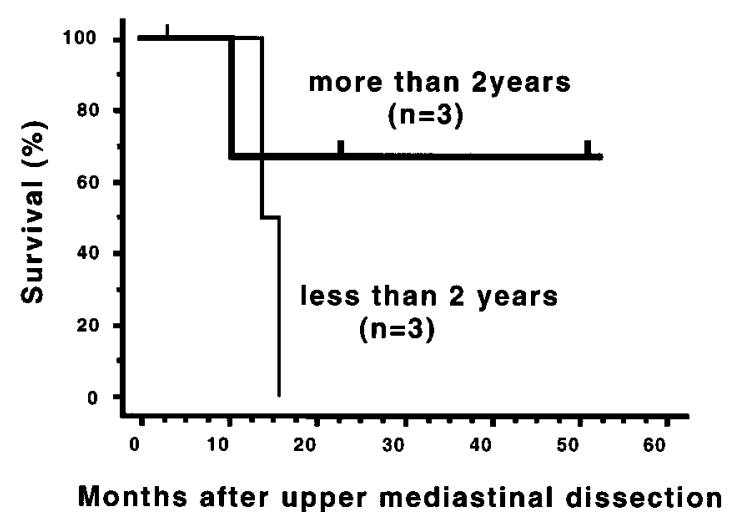

Fig 2. Survival curves after upper mediastinal dissection according to the length of time from the first operation to recurrence in patients undergoing complete resection. There was no significant difference between groups $(P=.36, \log$ rank test). The $95 \%$ confidence limits at 4 years in group labeled "more than 2 years" were 0.1 to 1.2 .

\section{REFERENCES}

1. Maassen W. The staging issue-problems: accuracy of mediastinoscopy. In: Delarue NC, Eschapasse H, editors. Lung cancer: international trends in general thoracic surgery. Philadelphia: WB Saunders; 1985: p. 42-53.

2. Hata E, Miyamoto H, Sakaguchi H, Futagawa T, Ikeda S, Asai K. The efficacy of systemic bilateral mediastinal dissection for survival and investigation into lymph node metastasis in patients of non small cell lung cancer (NSCLC). Lung Cancer 1997;18:S97.

3. Hirono T, Koike T, Eguchi S. Mediastinal lymph node dissection through median sternotomy-operative technique and results. Principal of Lung Cancer Surgery 1991;3:21-30.

4. Martini N, Bains M, Burt M, Zakowski M, McCormack P, Rusch $\mathrm{V}$, et al. Incidence of local recurrence and second primary tumors in resected stage I lung cancer. J Thorac Cardiovasc Surg 1995;109:120-129. 